\title{
Plethodontid salamander mitochondrial genomics: A parsimony evaluation of character conflict and implications for historical biogeography
}

J. Robert Macey ${ }^{1,2}$

${ }^{1}$ Department of Evolutionary Genomics, DOE Joint Genome Institute and Lawrence Berkeley

National Laboratory, 2800 Mitchell Drive, Walnut Creek, CA 94598

${ }^{2}$ Museum of Vertebrate Zoology, University of California, Berkeley, CA 94720

Running head: Plethodontid salamander mt-genomics

Correspondence to: J. Robert Macey, Department of Evolutionary Genomics, DOE Joint Genome Institute and Lawrence Berkeley National Laboratory, 2800 Mitchell Drive, Walnut Creek, CA 94598-1631; Phone: 925-296-5621; FAX: 925-296-5666; e-mail: jrmacey@lbl.gov 


\begin{abstract}
A new parsimony analysis of 27 complete mitochondrial genomic sequences is conducted to investigate the phylogenetic relationships of plethodontid salamanders. This analysis focuses on the amount of character conflict between phylogenetic trees recovered from newly conducted parsimony searches and the Bayesian and maximum likelihood topology reported by Mueller et al. (2004, PNAS, 101, 13820-13825). Strong support for Hemidactylium as the sister taxon to all other plethodontids is recovered from parsimony analyses. Plotting area relationships on the most parsimonious phylogenetic tree suggests that eastern North America is the origin of the family Plethodontidae supporting the "Out of Appalachia" hypothesis. A new taxonomy that recognizes clades recovered from phylogenetic analyses is proposed.
\end{abstract}

Key Words: Amphibia; Caudata; Plethodontidae; Phylogeny; Mitochondrial DNA; Genomics; Biogeography; Appalachia. 
1. Introduction

2. Methods

2.1. Specimen Information

2.2. Data Partitions

2.3. Phylogenetic Analysis

3. Results

3.1. Phylogenetic Relationships

3.2. Support for Bayesian and Maximum Likelihood Nodes in Parsimony Analyses of Subset Data Partitions

3.2. Statistical Evaluation of Alternative Hypotheses

4. Discussion

4.1. Character Conflict and the Phylogenetic position of Hemidactylium

4.2. Historical Biogeography

4.3. Taxonomic Recommendations

4.4. Phylogenetic Reconstruction in Our Time 


\section{Introduction}

The largest salamander family, Plethodontidae, is primarily restricted to the New World. Biologists have long hypothesized on the origins of this group. Three major centers of radiation are recognized in the family, (1) tropical America, (2) western North America, and (3) eastern North America. In addition, a small radiation is also found in southern Europe. Among these regions, eastern North America has been implicated as containing the root of the plethodontid clade (Wilder and Dunn, 1920), and termed the "Out of Appalachia" hypothesis (for review see Mueller et al., 2004).

Traditionally, the Plethodontidae is divided into two subfamilies Desmognathinae and Plethodontinae, with the later containing three tribes, Bolitoglossini, Hemidactyliini, and Plethodontini. The Desmognathinae is restricted to eastern North America, while the Plethodontinae is more complex in distribution. The tribe Bolitoglossini is primarily distributed in tropical America, but two genera occur in western North America (Batrachoseps and Hydromantes). In addition, in this group, a small radiation of Hydromantes species occur in southern Europe. The tribe Hemidactyliini is restricted to eastern North America. The tribe Plethodontini has a complex pattern of distribution between eastern and western North America, with several genera showing an east-west disjunct transcontinental occurrence.

Recently, Mueller et al. (2004) put forth a new phylogenetic study of the Plethodontidae based on complete mitochondrial genomic sequences. This study places a major focus on Bayesian and maximum likelihood phylogenetic tree reconstruction methods. Parsimony phylogenetic analyses conducted in Mueller et al. (2004), but not discussed in detail, identify a conflict with results generated from Bayesian and maximum likelihood phylogenetic 
reconstruction methods. The phylogenetic topologies derived from these different methods may have alternative biogeographic implications for plethodontid salamanders.

\section{Methods}

\subsection{Specimen Information}

Arranged in traditional taxonomy, the following published complete mitochondrial genomic sequences are analyzed, and GenBank accession numbers are presented. All samples from Mueller et al. (2004) have vouchers in the Museum of Vertebrate Zoology, University of California at Berkeley (MVZ).

Outgroup samples are: CRYPTOBRACHIDAE, Andrias davidianus; AJ492192 (Zhang et al., 2003a); HYNOBIIDAE, Ranodon sibiricus, AJ419960 (Zhang et al., 2003b); SALAMANDRIDAE, Salamandra luschani; AF154053 (Zardoya and Meyer, 2001); AMBYSTOMATIDAE, Ambystoma laterale, MVZ173468, AY728218 (Mueller et al., 2004); RHYACOTRITONIDAE, Rhyacotriton variegates, MVZ222581, AY728219 (Mueller et al., 2004).

In group samples from the Plethodontidae are: DesmognathinaE, Desmognathus fuscus, MVZ224931, AY728227; D. wrighti, MVZ222618, AY728225; Phaeognathus hubrichti, MVZ173507, AY728233. Plethodontinae, Bolitoglossini, Batrachoseps attenuatus, MVZ230761, AY728228; B. wrightorum, MVZ224902, AY728221; Bolitoglossa sp. nov., MVZ225875, AY728235; Hydromantes brunus, MVZ230641, AY728234; H. italicus, MVZ163996, AY728215; Nototriton abscondens, MVZ203743, AY728229; Oedipina poelzi, MVZ207128, AY728213; Thorius sp. nov., MVZ231444, AY728224. HEMIDACTYLIINI, Eurycea bislineata, MVZ225074, AY728217; Gyrinophilus porphyriticus, MVZ173504, AY728230; Hemidactylium scutatum, MVZ225077, AY728231; Pseudotriton rubber, 
MVZ220897, AY728220; Stereochilus marginatus, MVZ233227, AY728212. PLETHODONTINI, Aneides flavipunctatus, MVZ219973, AY728214; A. hardii, MVZ226110, AY728226; Ensatina eschscholtzii, MVZ236229, AY728216; Plethodon cinereus, MVZ225101, AY728232; P. elongates, MVZ220003, AY728223; P. petraeus, MVZ222650, AY728222 (all from Mueller et al., 2004).

\subsection{Data Partitions}

Mueller et al. (2004) had concern with base substitutional saturation. Therefore here the effect of removal of transitions and third codon positions are examined. Four data sets or data partitions are analyzed using parsimony. The mt-DNA total evidence data partition is defined as that containing all characters. The transversional data partition is defined as that using only transversions from all characters where $\mathrm{G}$ and $\mathrm{A}$ are converted to $\mathrm{R}$, and $\mathrm{C}$ and $\mathrm{T}$ are converted to $\mathrm{Y}$. The all data excluding third positions data partition is similar to the first data partition but does not include characters from third codon positions. Likewise, the transversional data excluding third positions partition is similar to the second data partition but does not include characters from third codon positions.

In addition, five data partitions are analyzed separately with parsimony to evaluate support from decay indices for nodes found in Bayesian and maximum likelihood phylogenetic trees. These data partitions are, tRNA genes, rRNA genes, first codon positions, second codon positions and third codon positions of protein coding genes, and are referred to as subset data partitions to avoid confusion with the data partitions described above. These character classes were selected based on functional properties. Both all data and transversional anlyses were run on the subset data partitions. 


\subsection{Phylogenetic Analysis}

The DNA sequence alignment of Mueller et al. (2004) is used with the same exclusion set, and is available in TreeBASE (http://www.treebase.org/treebase/, study accession number S1139, matrix accession number M1957). This alignment contains 15,852 sites with 1,810 excluded, yielding 14,042 base positions for potential phylogenetic analysis.

Phylogenetic trees were inferred by parsimony using PAUP* beta version $4.0 \mathrm{~b} 8$ (Swofford, 2001) with heuristic searches featuring 100 random addition of taxa. Bootstrap resampling (Felsenstein, 1985a) was applied to assess support for individual nodes using 1000 heuristic searches featuring 100 random additions of taxa.

Decay indices (= "branch support" of Bremer, 1994) were calculated for all internal branches using searches that retained suboptimal nodes. When branches did not exist in the tree derived from the mt-DNA total evidence data, constrained analyses were conducted using MacClade (Maddison and Maddison, 2001) as input trees to PAUP* (Swofford, 2001) undergoing suboptimal searches. These values throughout the paper are recorded as negative numbers or "cost values".

When evaluating support with the five subset data partitions discussed above for nodes found in Bayesian and maximum likelihood phylogenetic trees that are not recovered in the parsimony mt-DNA total evidence tree, a similar approach was taken. In this case, positive numbers represent presence of the Bayesian and maximum likelihood node in a parsimony search of the data from that subset data partition and a negative number depicts the cost in steps to find a tree with that node. 
Alternative phylogenetic hypotheses were tested using the most parsimonious phylogenetic topologies compatible with each. To find the most parsimonious tree(s) compatible with a particular phylogenetic hypothesis, topologies were first constructed using MacClade (Maddison and Maddison, 2001), providing input as constraint trees to PAUP* (Swofford, 2001) for subsequent searches. Wilcoxon signed-ranks tests (Templeton, 1983) were used to examine statistical significance of the shortest tree relative to alternative hypotheses. This test determines whether the most parsimonious tree is significantly shorter than an alternative or whether their differences in length are statistically indistinguishable. Wilcoxon signed-ranks tests were conducted as two-tailed tests (Felsenstein, 1985b). Tests were conducted using PAUP* (Swofford, 2001), which incorporates a correction for tied ranks.

\section{Results}

\subsection{Phylogenetic Relationships}

Hypotheses of phylogenetic relationships among plethodontid salamanders derived via parsimony with all characters included are depicted in figure 1A (ie., mt-DNA total evidence tree). A very similar topology is acquired in a parsimony analysis of transversional data (Fig. 1B). These topologies are markedly different than that acquired by Mueller et al. (2004) applying Bayesian and maximum likelihood criteria for tree reconstruction (Fig. 1C).

Shared among these trees are: the outgroup structure, nodes 1-4; monophyly of Batrachoseps, node 7; monophyly of Oedipina, Nototriton, Bolitoglossa and Thorius (=tropical salamander clade), node 8; monophyly of Oedipina and Nototriton, node 9; monophyly of Eurycea, Pseudotriton, Gyrinophilus and Steriochilus (=Hemidactyliini not including 
Hemidactylium, here termed the Eurycea clade), node 12; monophyly of Pseudotriton, Gyrinophilus and Steriochilus, node 13; monophyly of Plethodon, Phaeognathus, Desmongnathus, Aneides, Hydromantes, and Ensatina (=Desmognathinae and Plethodontini plus Hydromantes), node 15; monophyly of Plethodon, node 16; monophyly of eastern Plethodon (P. cinereus and P. petraeus), node 17; monophyly of Phaeognathus and Desmognathus (=Desmognathinae), node 19; monophyly of Desmognathus, node 20; monophyly of Aneides, node 22; and monophyly of Hydromantes, node 24. An additional node is shared between the mt-DNA total evidence data partition and the Bayesian-maximum likelihood tree, monophyly of Phaeognathus, Desmognathus, Aneides, Hydromantes, and Ensatina, node 18. In summary, the major clades that are in total agreement are the (A) tropical salamander clade, node 8, (B) Eurycea clade, node 12, (C) Desmognathinae-Plethodontini plus Hydromantes clade, node 15, and (D) Desmognathinae clade, node 19.

In comparing the mt-DNA total evidence parsimony tree with the Bayesian-maximum likelihood tree, seven nodes are in conflict (nodes 5, 6, 10, 11, 14, 21, and 23, Fig. 1A; nodes a-g Fig. 1C). The number of extra steps required to individually constrain nodes a-g of the Bayesian-maximum likelihood tree in mt-DNA total evidence parsimony analyses is costly, ranging from 15 to 76 steps per node (Fig. 1C).

Node 5, the monophyly of all plethodontids to the exclusion of Hemidactylium appears to be well supported in parsimony analyses (mt-DNA total evidence bootstrap 89\%, decay index 28; transversional data 99\%, decay index 43). Removal of third positions from both all of the data and the transversional data does not result in the destruction of this node (Table 1).

Node 6, the monophyly of Batrachoseps and the tropical salamanders (=Bolitoglossini not including Hydromantes) receives moderate support (bootstrap 84\%, decay index 18) from the 
mt-DNA total evidence data, and is present in one of two equally parsimonious trees in analysis of the transversional data. Only the removal of third positions from the transversional data partition results in destruction of this branch, but at a cost of 4 steps the branch can be recovered (Table 1).

Node 10, the monophyly of Bolitoglossa and Thorius, receives strong support from both the mt-DNA total evidence and transversional data (mt-DNA total evidence bootstrap 100\%, decay index 68; transversional data bootstrap 89\%, decay index 19). Removal of third positions from all of the data results in a fairly high decay index of 21 , and from the transversional data does not result in the destruction of this node (Table 1). In addition, to acquire the phylogenetic arrangement within the tropical salamander clade observed from the Bayesian-maximum likelihood analyses a cost of 76 steps is required.

Node 11, monophyly of the Eurycea clade plus the clade containing Desmognathinae and Plethodontini is weakly supported in the mt-DNA total evidence and transversional analyses (mtDNA total evidence bootstrap 56\%, decay index 4; transversional data bootstrap 67\%, decay index 7). Removal of third positions from the mt-DNA total evidence data results in a topology that is 15 steps shorter than the one containing this arrangement, and removal of third positions from the transversional data finds a tree 1 step shorter.

Node 14, monophyly of Gyrinophilus and Stereochilus receives fairly good support from the total evidence data (bootstrap $87 \%$, decay index 25) but is only weakly supported by the transversional data (bootstrap 53\%, decay index 1). Interestingly, removal of third positions from both all of the data and the transversional data does not result in the destruction of this node. 
Node 21, monophyly of Aneides, Ensatina, and Hydromantes is very weakly supported in the mt-DNA total evidence data (decay index 2). All other analyses do not recover this branch.

Node 23, monophyly of Ensatina and Hydromantes is a branch with real conflict. In the mt-DNA total evidence analysis, this branch receives weak boostrap support of 53\% but has a decay index of 15. Analysis of the transversional data obtains branch "g" (monophyly of Aneides and Hydromantes, Fig. 1B,C) of the Bayesian-maximum likelihood tree, and it costs 15 steps to obtain a tree containing node 23. Removal of third positions from the mt-DNA total evidence data requires 8 extra steps to obtain a tree with node 23 , and from the transversional data this node is present in only one of several equally most parsimonious solutions.

Among the seven nodes incongruent between the mt-DNA total evidence parsimony analysis and the phylogenetic tree topology recovered from Bayesian-maximum likelihood reconstruction methods, a surprisingly small amount of character conflict is attributable to transitional changes and third positions. Nodes 5, 10, and 14 are recovered in all parsimony analyses. Node 6 is not recovered in analyses including transversions only but few extra steps are required to obtain this branch. Node 11 is weak and affected by exclusion of third positions. Node 21 is extremely weak. Node 23 is weakly supported but appears to show some degree of conflict between data partitions.

\subsection{Support for Bayesian and Maximum Likelihood Nodes in Parsimony Analyses of Subset}

\section{Data Partitions}

In order to examine these data further for any substantial phylogenetic signal of nodes "ag" (Fig. 1C) found in Bayesian and maximum likelihood analyses but not recovered in the parsimony analysis of the mt-DNA total evidence data, a decay analysis was conducted of subset 
data partitions (Table 2). Parsimony analysis of tRNA genes, rRNA genes, first codon positions, second codon positions and third codon positions of protein coding genes, each separately, recover little support for nodes "a-g" in figure $1 \mathrm{C}$.

Nodes "d" (monophyly of Hemidactylium and Batrachoseps), "e" (monophyly of Bolitoglossa, Oedipina and Nototriton) and "f" (monophyly of Ensatina, Phaeognathus and Desmognathus) never are recovered in parsimony analyses of these data partitions even when only analyzing transversions.

Node "a", depicting monophyly of the Eurycea clade, Hemidactylium, Batrachoseps and the tropical salamanders, when present, collapses in 1-3 steps. In contrast, when node "a" is not present it can be costly, up to 45 and 46 steps in analyses of third positions and third position transversions, respectively.

Node "b", monophyly of Gyrinophilus and Stereochilus, shows a similar pattern of little support for this node except for third position transversions with a decay index of 6 . In contrast, analysis of all third position changes costs 20 steps to recover node "b".

Node "f", monophyly of Ensatina, Phaeognathus and Desmognathus, was only recovered in alaysis of transversions from rRNA genes with a decay index of 2. Again, it can be costly to recover this node with 24 and 26 extra steps required in analyses of third positions and third position transversions, respectively.

Node "g", monophyly of Hydromantes and Aneides, shows real conflict. On one hand analysis of third positions and third position transversions actually recover this node with decay indeces of 9 and 10, respectively. On the other hand all analyses of other data partitions do not recover this node and it can be costly, up to 18 extra steps in analysis of first positions. 
Of the 70 parsimony analyses run on the five subset data partitions, only 12 recovered nodes present in the Bayesian and maximum likelihood tree (Table 2). Only three of these had decay indices above 3 steps. Among these 70 parsimony analyses, over half require more than 3 extra steps to force the presence of nodes "a-g" recovered from Bayesian and maximum likelihood analyses. In general, this is most costly when analyzing third positions or third position transversions.

\subsection{Statistical Evaluation of Alternative Hypotheses}

To further evaluate which data sets are in conflict the Wilcoxon signed-ranks test (Felsenstein, 1985b; Templeton, 1983) is applied to parsimoniously compare alternative tree toplogies.

(1) The tree topology derived from the Bayesian-maximum likelihood analysis has seven nodes not present in the tree derived from the mt-DNA total evidence parsimony analysis. The Bayesian-maximum likelihood tree requires 153 extra steps using the mt-DNA total evidence data and is rejected in favor of the unconstrained shortest tree (46619 steps, Fig. 1A; $n=1557, \mathrm{Z}$ $=-3.5662, P<0.0004)$.

(2) The Bayesian-maximum likelihood tree requires 103 extra steps using the transversional data and is rejected in favor of the unconstrained shortest tree (18757 steps, Fig. $1 \mathrm{~B} ; \mathrm{n}=702, \mathrm{Z}=-3.7147, P<0.0002 ; \mathrm{n}=710, \mathrm{Z}=-3.6689, P<0.0002)$.

(3) The Bayesian-maximum likelihood tree requires 77 extra steps using the mt-DNA total evidence data excluding 3rd positions and is rejected in favor of the unconstrained shortest tree (20606 steps; $\mathrm{n}=718, \mathrm{Z}=-2.6447, P<0.0082)$. 
(4) The Bayesian-maximum likelihood tree requires 20 extra steps using the transversional data excluding 3rd positions and is not rejected in favor of the unconstrained shortest tree (6209 steps; $\mathrm{n}=194, \mathrm{Z}=-1.3499, P<0.1771 ; \mathrm{n}=195, \mathrm{Z}=-1.3802, P<0.1675) ; \mathrm{n}$ $=194, \mathrm{Z}=-1.3752, P<0.1691) ; \mathrm{n}=185, \mathrm{Z}=-1.4142, P<0.1573)$.

Of biogeographic importance is node 5 which places Hemidactylium as the sister taxon to all other plethodontid salamanders, suggestive of a deep history in eastern North America. This node receives high bootstrap support and is present in parsimony analyses of all data partitions. The shortest alternative tree that does not show all plethodontid salamanders as monophyletic with the exclusion of Hemidactylium requires 28 extra steps applying the mt-DNA total evidence data and is not rejected in favor of the unconstrained shortest tree $(46,647$ steps, $n=1166, Z=-$ 0.7983, $P<0.4247$ ); and 43 extra steps applying the transversional data and is rejected in favor of the unconstrained shortest tree $(18,515$ steps, $\mathrm{n}=299, \mathrm{Z}=-2.4868, P<0.0129 ; \mathrm{n}=291, \mathrm{Z}=-$ 2.5207, $P<0.0117 ; \mathrm{n}=249, \mathrm{Z}=-2.7250, P<0.0064 ; \mathrm{n}=361, \mathrm{Z}=-2.1977, P<0.0280 ; \mathrm{n}=$ 291, $\mathrm{Z}=-2.5207, P<0.0117 ; \mathrm{n}=179, \mathrm{Z}=-3.2140, P<0.0013)$.

\section{Discussion}

\subsection{Character Conflict and the Phylogenetic Position of Hemidactylium}

Parsimony analysis of alternative data partitions are statistically incompatible with the tree topology produced from Bayesian-maximum likelihood analyses except when both transitions and third positions are simultaneously excluded, yet Hemidactylium remains the sister taxon to all other plethodontids when third positions are removed. This suggests that third position transversions contribute significantly to the discrepancy in phylogenetic results among methods. 
When parsimony analyses are conducted on subset data partitions of tRNA genes, rRNA genes, first codon positions, second codon positions and third codon positions of protein coding genes, little support is recovered for nodes "a-g" present in the Bayesian and maximum likelihood tree (Fig. 1C). Again, the most striking conflicts come from costly numbers of extra steps required to recover most of these nodes when analyzing third positions. Alternatively, a general pattern of low decay indices from 1 to 3 steps are observed when analysis of a data partition actually recovers one of nodes "a-g".

Often third codon positions are considered less informative than first and second codon positions because they are relatively faster evolving. Because mitochondrial proteins are transmembrane proteins with very stringent hydrophobic regions to accommodate ionic movement, selection for charged amino acids in ionic channels, and uncharged amino acid positions in hydrophilic tails of proteins can be strong. Third position substitutions usually do not result in amino acid changes. When they do it is restricted to transversional changes that rarely change hydrophobicity and therefore are presumably more free from selection pressures driven by hydrophobicity. Third position transversions are the more conservative class of these substitutions and may in fact be one of the more reliable classes of characters in the mitochondrial genome because of this apparent lack of selection.

All parsimony analyses recover Hemidactylium as the sister taxon to the remaining Plethodontidae as run in table 1 (node 5). This node receives strong bootstrap support from analysis of the mt-DNA total evidence data partition and statistical support from analysis of transversions. There is no support for node "d" in the Bayesian-maximum likelihood tree uniting Hemidactylium with Batrachoseps in parsimony analyses of subset data partitions. Hence, there is considerable support for a basal position of Hemidactylium among plethodontid salamanders. 


\subsection{Historical Biogeography}

Plethodontid salamanders have long been considered to have their origins in the greater Appalachian mountain region of eastern North America (Wilder and Dunn, 1920). This hypothesis is mainly based on the vast richness of species numbers, diversity of habitats occupied, and occurrence of numerous life histories. Subsequent speciation and dispersal from eastern North America to western North America, tropical America, and southern Europe has been termed the "Out of Appalachia" hypothesis.

The phylogenetic tree derived here can be used to examine area relationships (Fig. 2). When areas are plotted on the shortest tree based on the mt-DNA total evidence data, eastern North America is suggested to be the ancestral area for the Plethodontidae. The fact that Hemidactylium is likely to represent the sister taxon to the remaining plethodontids indicates that at least the basal split in the family involved eastern North America. If Pleistocene glaciated regions of the distribution of Hemidactylium are eliminated, its area of endemism is refined in the broad sense to the ancient plateau region of greater Appalachia (including the Ozark and Ouachita mountains). Both the ancestral reconstruction presented in figure 2 and the basal position of Hemidactylium support a root of the plethodontid clade in eastern North America, and therefore are consistent with the "Out of Appalachia" hypothesis.

\subsection{Taxonomic Recommendations}

Results from phylogenetic analyses presented in Mueller et al. (2004) and the phylogenetic analyses presented here do not conform to currently recognized taxonomy. Results from the parsimony analysis require fewer taxonomic changes because Batrachoseps is grouped 
with the tropical salamander clade which represents the traditional Bolitoglossini with only the exclusion of Hydromantes. All other major groups are equally disrupted by the Bayesianmaximum likelihood and parsimony analyses. In addition, major groupings discovered in parsimony analyses are also recovered with nuclear DNA sequence data (Chippindale et al., 2004).

Here, based on the criterion of monophyly, it is suggested that recognition of the subfamily Desmognathinae be discontinued and this group be placed in the Plethodontinae along with Hydromantes. The Plethodontinae is then further restricted to only contain the members of the Plethodontini in the former Plethodontinae. Hence, in the sampling conducted in this study the Plethodontinae contains the monophyletic grouping of Plethodon, Phaeognathus, Desmognathus, Aneides, Ensatina, and Hydromantes, (node 15 in Fig. 1).

Hemidactylium is placed in its own subfamily, the Hemidactylinae (first recognized as a family by Hallowell, 1856, Proc. Acad. Nat. Sci. Philadelphia, 8: 11, type genus Hemidactylium Tschudi, 1838; see Frost, 2004), because it is excluded from all other Plethodontidae by node 5 (Fig. 1A,B). The remaining taxa previously assigned to the Hemidactyliini are placed in a separate subfamily, named in Chippindale et al. (2004), containing Eurycea, Stereochilus, Gyrinophilus, and Pseudotriton, (node 12, Fig. 1). The Bolitoglossini is elevated to subfamilial status, the Bolitoglossinae (first recognized as a family by Hallowell, 1856, Proc. Acad. Nat. Sci. Philadelphia, 8:11, type genus Bolitoglossa Duméril, Bibron, and Duméril, 1854; see Frost, 2004), (node 6, Fig. 1A ), and retains its original content with only the exclusion of Hydromates, here placed in the Plethodontinae. Hence, the family Plethodontidae now contains four subfamilies, the Plethodontinae, Hemidactylinae, Bolitoglossinae, and the Eurycea clade named 
in Chippindale et al. (2004). Remarkably, the taxonomy presented here is independently derived and advocated by Chippindale et al. (2004).

\subsection{Phylogenetic Reconstruction in Our Time}

Phylogenetic reconstruction has increasingly become computer driven. Debate among researchers as to the extent the character needs to be preserved in phylogenetic analyses is not new, but rather more intense than ever. This is analogous to conducting math with a calculator and saying you understand math. It is this authors view that much more care needs to be given to what phylogenetic methods are actually doing with the characters in a data set than presently respected among the community. This is particularly true with molecular data, as numerous researchers doing molecular phylogenetics do not really understand the biochemistry behind the characters they are collecting and analyzing for phylogenetic inference.

\section{ACKNOWLEDGEMENTS}

This work is LBNL-56662 and was performed under the auspices of the U.S. Department of Energy, Office of Biological and Environmental Research, under contract No. DE-AC0376SF00098 with the University of California, Lawrence Berkeley National Laboratory. I thank Paul Chippindale for providing a copy of his unpublished manuscript, and Arnold Kluge and three anonymous reviewers for providing useful suggestions to expand this manuscript. 


\section{REFERENCES}

Bremer, K. (1994). Branch support and tree stability. Cladistics 10, 295-304.

Chippindale, P. T., Bonett, R. M., Baldwin, A. S., and Wiens, J. J. (2004). Phylogenetic evidence for a major reversal of life history evolution in plethodontid salamanders. Evol. (in press).

Felsenstein, J. (1985a). Confidence limits on phylogenies: An approach using the bootstrap. Evol. 39, 783-791.

Felsenstein, J. (1985b). Confidence limits on phylogenies with a molecular clock. Syst. Zool. 34, $152-161$.

Frost, D. R. (2004). Amphibian Species of the World: an Online Reference. Version 3.0 (22 August, 2004). Electronic Database accessible at http://research.amnh.org/herpetology/amphibia/index.php. American Museum of Natural History, New York, USA

Maddison, W. P., and Maddison, D. R. (2001). "MacClade, Analysis of Phylogeny and Character Evolution, Version 4.03," Sinauer, Sunderland, MA.

Mueller, R. L., Macey, J. R., Jaekel, M., Wake, D. B., and Boore, J. L. (2004). Morphological homoplasy, life history evolution, and historical biogeography of plethodontid salamanders: Novel insights from complete mitochondrial genome sequences. Proc. Natl. Acad. Sci. 101, 13820-13825.

Swofford D. L. (2001). "PAUP* Phylogenetic Analysis Using Parsimony (*and Other Methods), Beta Version 4.0b8," Sinauer, Sunderland, MA. 
Templeton, A. R. (1983). Phylogenetic inference from restriction endonuclease cleavage site maps with particular reference to the evolution of humans and the apes. Evol. 37, 221244.

Wilder, I. W., and Dunn, E. R., (1920). The correlation of lunglessness in salamanders with a mountain brook habitat. Copeia 1920, 63-68.

Zardoya, R., Meyer, A., 2001. On the origin of and phylogenetic relationships among living amphibians. Proc. Natl. Acad. Sci. USA 98, 7380-7383.

Zhang, P., Chen, Y. Q., Liu, Y. F., Zhou, H., Qu, L. H., 2003a. The complete mitochondrial genome of the Chinese giant salamander, Andrias davidianus (Amphibia: Caudata). Gene 311, 93-98.

Zhang, P., Chen, Y. Q., Zhou, H., Wang, X. L., Qu, L. H., 2003b. The complete mitochondrial genome of a relic salamander, Ranodon sibiricus (Amphibia: Caudata) and implications for amphibian phylogeny. Mol. Phylogenet. Evol. 28, 620-626. 
Table 1

Decay analysis among alternative data partitions as plotted on the phylogenetic tree derived from mt-DNA total evidence

\begin{tabular}{lllllllllllllllllllllllll}
\hline Node No. & 1 & 2 & 3 & 4 & 5 & 6 & 7 & 8 & 9 & 10 & 11 & 12 & 13 & 14 & 15 & 16 & 17 & 18 & 19 & 20 & 21 & 22 & 23 & 24 \\
\hline All data & 66 & 45 & 7 & 176 & 28 & 18 & 102 & 89 & 118 & 68 & 4 & 103 & 204 & 25 & 86 & 15 & 360 & 2 & 36 & 163 & 2 & 233 & 15 & 140 \\
No 3rd positions & 54 & 16 & 18 & 148 & 2 & 2 & 58 & 47 & 82 & 21 & $\mathbf{- 1 5}$ & 60 & 120 & 4 & 8 & 6 & 263 & 1 & $\mathbf{- 6}$ & 75 & $\mathbf{- 1 3}$ & 102 & $\mathbf{- 8}$ & 110 \\
TV, all data & 54 & 16 & 19 & 145 & 43 & 0 & 75 & 11 & 109 & 19 & 7 & 80 & 123 & 1 & 66 & 17 & 305 & $\mathbf{- 2 4}$ & 7 & 113 & $\mathbf{- 7}$ & 131 & $\mathbf{- 1 5}$ & 172 \\
TV, no 3rd positions & 50 & 15 & 14 & 81 & 1 & $\mathbf{- 4}$ & 28 & 18 & 58 & 2 & $\mathbf{- 1}$ & 17 & 49 & 2 & 7 & 7 & 152 & $\mathbf{- 6}$ & 4 & 45 & $\mathbf{- 2}$ & 41 & 0 & 60 \\
\hline
\end{tabular}

Note: Nodes are labeled as in figure 1A. TV $=$ transversions. Negative numbers in bold are cost values and decay indices appear in plain type. 
Table 2. Parsimony decay analysis among subset data partitions as plotted on the phylogenetic tree derived from Bayesian and maximum likelihood phylogenetic reconstruction methods

\begin{tabular}{lllllllll}
\hline Character & $\begin{array}{l}\text { Node } \\
\text { Class }\end{array}$ & $\begin{array}{l}\text { Node } \\
\text { B }\end{array}$ & $\begin{array}{l}\text { Node } \\
\text { C }\end{array}$ & $\begin{array}{l}\text { Node } \\
\text { D }\end{array}$ & $\begin{array}{l}\text { Node } \\
\text { E }\end{array}$ & $\begin{array}{l}\text { Node } \\
\text { F }\end{array}$ & $\begin{array}{l}\text { Node } \\
\text { G }\end{array}$ & $\begin{array}{l}\text { No. of } \\
\text { Informative } \\
\text { Sites }\end{array}$ \\
\hline All data & -28 & -25 & -28 & -28 & -76 & -22 & -15 & 7056 \\
tRNAs & $\mathbf{3}$ & -5 & -1 & -1 & -12 & -11 & 0 & 657 \\
rRNAs & $\mathbf{1}$ & -2 & -3 & -3 & -14 & -2 & -1 & 676 \\
$\begin{array}{l}\text { 1st } \\
\text { positions }\end{array}$ & -8 & $\mathbf{1}$ & -14 & -11 & -9 & -3 & -18 & 1601 \\
$\begin{array}{l}\text { 2nd } \\
\text { positions }\end{array}$ & -3 & $\mathbf{2}$ & -4 & -3 & -4 & -6 & -2 & 751 \\
$\begin{array}{l}\text { 3rd } \\
\text { positions }\end{array}$ & -45 & -20 & -13 & -18 & -30 & -24 & $\mathbf{9}$ & 3371 \\
$\begin{array}{l}\text { TV all } \\
\text { data }\end{array}$ & -43 & -1 & -44 & -43 & -19 & -17 & $\mathbf{7}$ & 3982 \\
$\begin{array}{l}\text { TV } \\
\text { tRNAs }\end{array}$ & $\mathbf{3}$ & $\mathbf{1}$ & -2 & -1 & -4 & -7 & -2 & 173 \\
$\begin{array}{l}\text { TV } \\
\text { rRNAs }\end{array}$ & $\mathbf{1}$ & 0 & -4 & -3 & -5 & $\mathbf{2}$ & -2 & 308 \\
$\begin{array}{l}\text { TV 1st } \\
\text { positions }\end{array}$ & -5 & -4 & -6 & -5 & 0 & -1 & -5 & 891 \\
$\begin{array}{l}\text { TV 2nd } \\
\text { positions }\end{array}$ & $\mathbf{1}$ & $\mathbf{1}$ & -2 & -1 & 0 & -2 & -4 & 279 \\
$\begin{array}{l}\text { TV 3rd } \\
\text { positions }\end{array}$ & -46 & $\mathbf{6}$ & -37 & -45 & -18 & -26 & $\mathbf{1 0}$ & 2331 \\
\hline
\end{tabular}

Note: Nodes are labeled as in figure 1C. Numbers in bold are decay indices and negative numbers are cost values. 


\section{FIGURE LEGENDS}

FIG. 1. A). The single most parsimonious tree produced from analysis of the mt-DNA total evidence data partition containing 14,042 aligned nucleotide positions with 7056 phylogenetically informative sites. The tree has a length of 46,619 steps. Bootstrap values appear above and decay indices below branches. Nodes are numbered in bold below branches. B) Strict consensus of two equally parsimonious trees produced from analysis of the transversion only data partition containing 14,042 aligned nucleotide positions with 3982 phylogenetically informative sites. The tree has a length of 18,472 steps. Bootstrap values appear above and decay indices below branches. Nodes are numbered in bold below branches as in A, except for the three branches along the backbone of the tree not discovered in the analysis of the mt-DNA total evidence data partition. A single node labeled " $\mathrm{g}$ " is represented in the Bayesian and maximum likelihood tree of Mueller et al. (2004). C). Bayesian and maximum likelihood tree of Mueller et al. (2004). This tree has a length of 46,772 steps using the parsimony mt-DNA total evidence data, overall costing an additional 153 steps (see text). The seven nodes in conflict with the parsimony mt-DNA total evidence phylogenetic hypothesis are indicated as "a-g" with negative numbers referring to the number of steps required to find a tree with each particular branch in constrained phylogenetic searches. In Mueller et al. (2004) node "a" contained a bootstrap of $61 \%$ from parsimony analysis excluding third positions. In fact, parsimony analysis excluding third positions places Hemidactylium as the sister taxon to all other plethodontid salamanders (see table 1) and node "a" is not present in that tree. 
FIG. 2. Area cladogram of the Plethodontidae based on the most parsimonious tree derived from the mt-DNA total evidence data partition (Fig. 1A). Note that eastern North American taxa span the root of the tree and are suggested to be representative of the ancestral distribution (blue). An equivocal (hatching) dispersal is suggested to extend to either western North America (red) or tropical America (orange). Other dispersal events extend to western North America and Europe (red and black respectively). Major clades identified in this study and Mueller et al. (2004) are designated by letters where $\mathrm{A}=$ Hemidactylinae, $\mathrm{B}=$ Bolitoglossinae, $\mathrm{C}=$ the Eurycea clade named in Chippindale et al. (2004), and D = Plethodontinae. 

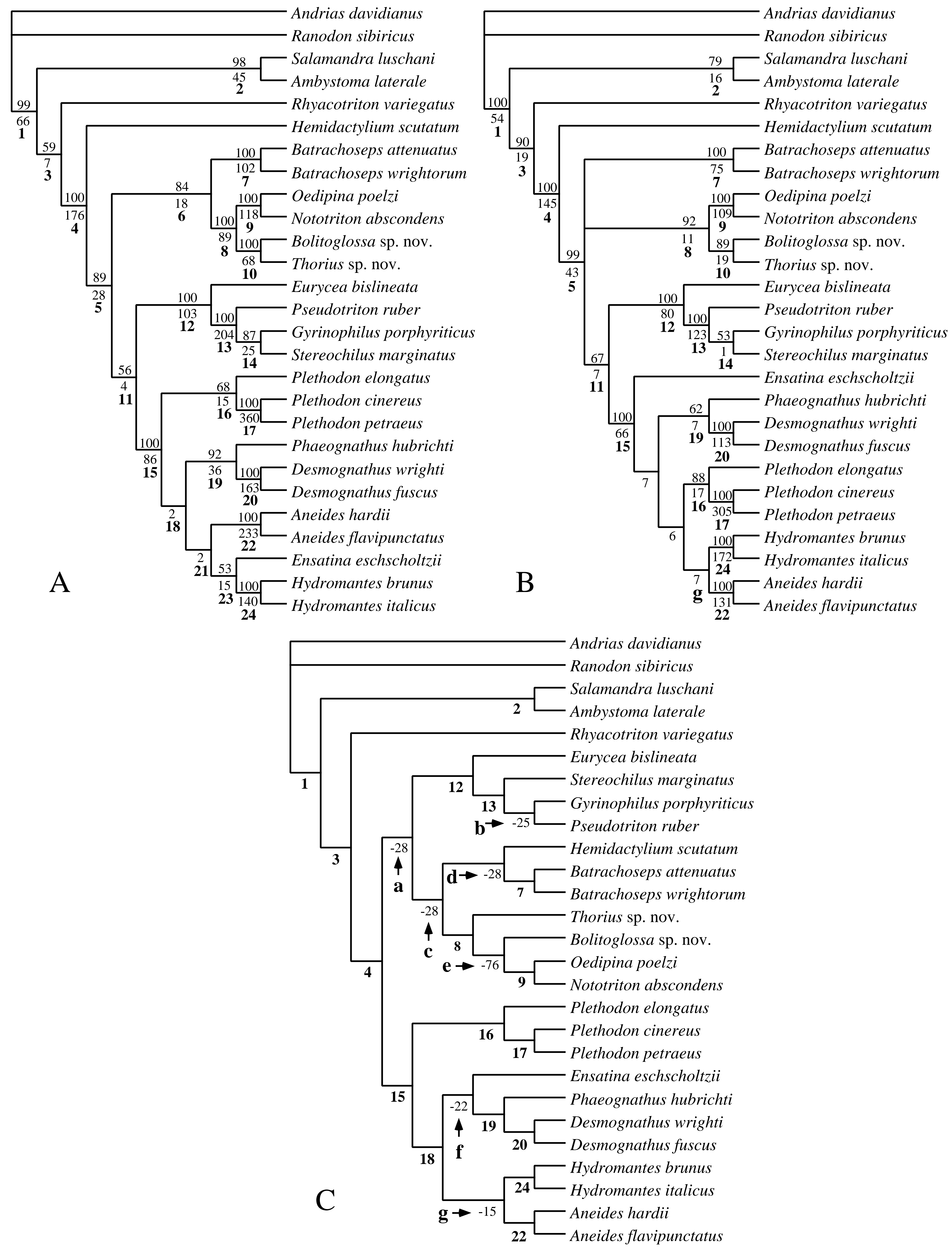


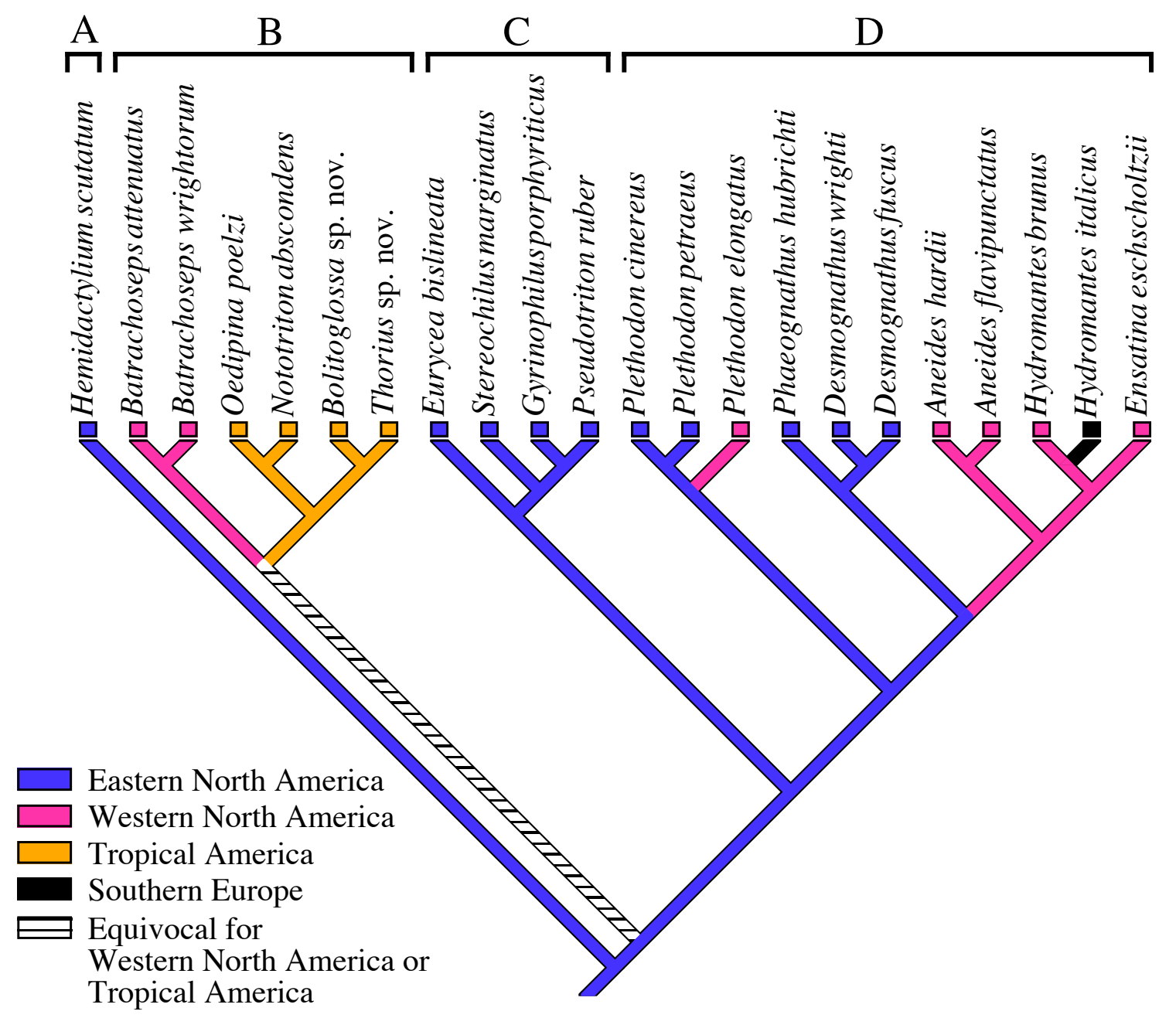

